\title{
A drug delivery system in controling the spread of the disease
}

\author{
Tong Zheng Jiang \\ North China Electric Power University \\ 689, Huadian Road, Baoding, Hebei Province 071003 \\ P.R.China \\ jiangtongzheng_001@163.com
}

Keywords: Graph theory; Monte Carlo algorithm; Global optimization model; Site selection problem

\begin{abstract}
In order to better control the spread of the disease, we should build a drug delivery system when the Ebola spread throughout the West Africa. We proposed that build up medical service centers to deliver drugs and vaccines to isolated regions. According to graph theory, the minimum number of the centers is set to seven under the conditions of the total area $428966 \mathrm{~km}^{2}$ of three regions being Guinea, Liberia and Sierra Leone, and the effective radius of single center is $140 \mathrm{~km}$. Afterwards, we assume that there are 50 isolated regions and the severity of epidemic region would take effect on drug demand, which refers to the Monte Carlo algorithm. Considering the cost and loss of the drug delivery, we select coordinates according to city site and develop constraint inequalities. Also, we use the global optimization model to deal with such a site selection problem. Under this situation, the optimal number of service centers is obtained as 10 after solving the mode. Thus, it's important to build a drug delivery system to control the propagation of Ebola.
\end{abstract}

\section{Introduction}

Considering the actual epidemic prevention program, we have established a number of isolated regions so far and most infected people are gathered here. As to the special transportation and preservation methods for vaccine and drug, we first establish the medical service center to distribute drugs and inject vaccine, in which we ignore the effects of topography and population density. Later, we use the basic knowledge of graph theory [1,2] to establish the coordinate system in the epidemic outbreak of West Africa. After calculation, we get the minimum number of the centers is set to seven under the conditions of the total area $428966 \mathrm{~km}^{2}$ of three regions being Guinea, Liberia and Sierra Leone [3], and the effective radius of single center is $140 \mathrm{~km}$.

Considering the different severity of the epidemic, different demand for drugs and different amount of drug transport cost, we then establish global optimization medical [4,5] to obtain the optimal number of service center and the best location [6,7]. Under this situation, the optimal number of service centers is obtained as 10 after solving the mode.

We further optimized and evaluated the model. In the initial model, the capacities of health care centers are equal. In optimization, we distribute different weights of medical service center depending on the severity of epidemic of different locations to make an impact on the maximum capacity of the service center. Then we substitute the weights into the model for solving the objective function again. We found the target optimization value is larger than before, and the outputs of the coordinate position are also more reasonable than previously obtained.

\section{System establishment}

In our delivery systems, we have separated the people who require the help into 3 categories. Most urgently, there are those who have already become infected with Ebola virus and people close to them. Next are the medical and support staff who treat patients, and those who handle the corpses. A case can 
be made that protection should also be offered to key domestic government workers and others providing essential logistical support.

Taking the actual vaccination program into account, it has established a number of isolated regions, where these infected people are concentrated. As for the high cost for the medicines and the high demand for storage conditions, as well as losses in the longer transport, in the international epidemic, we usually consider about establishing health care centers to issue medicines and vaccinations to quarantine. We are going to set the maximum coverage radius service stations, to use the basic knowledge of graph theory, to establish the coordinates, to consider about the needs for different regions of the severity, of the epidemic on drugs the amount, of the loss amount, of drug transportation and transportation costs and other conditions, the establishment of global optimization model is used to calculate the best location to get the minimum number and service center.

By querying the data, the total area of the three countries focused on the outbreak of the epidemic in West Africa is $428966 \mathrm{~km}^{2}$, totally including 56 cities. According to the fact, we can reasonably make assumptions that:

1, Every single service area covers a radius of $140 \mathrm{~km}$, and the area of each service in the maximum situation is $s=\pi \cdot R^{2}$. The number of required service areas at least is: $T_{\min }=\left\lceil\frac{S}{s}\right\rceil=7$,

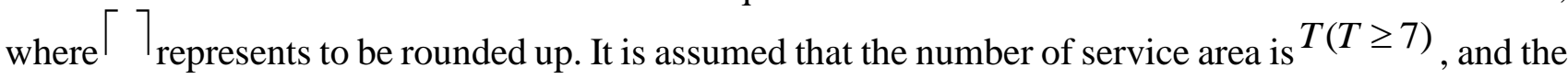
service area coordinates respectively are $P_{j}\left(x_{j}, y_{j}\right)(j=1,2, \cdots, T)$;

2 , Now quarantine settings are completed, which are located in 56 cities. We use Monte Carlo algorithm to randomly generate 50 coordinate points [8], which represent 50 quarantine. The random distribution of the 50 quarantine coordinates are $Q_{i}\left(x_{i}, y_{i}\right)$. At the same time, according to the geographical distribution of the epidemic map, Sierra Leone, as a result of the worst center for the disease [9]. For simplifying the calculation, the areas of those three regions can be approximated as an isosceles right triangle with sides of length 1 unit and the coordinates of the center of Sierra Leone can be approximated as: $(0.5,0.5)$

Due to the closer distances from the center of the epidemic area, outbreak becomes more of a problem, the more quantity $w_{i},(i=1,2, \cdots, 50)$ of the isolation required. Thus, according to the isolation of different geographical location, we set up the following inequality:

$$
\left\{\begin{array}{l}
\left(x_{i}-0.5\right)^{2}+\left(y_{i}-0.5\right)^{2}<k^{2} \\
\sum_{i=1}^{50} w_{i}=A
\end{array}\right.
$$

Among them, $k$ is the judgment for the spread degree of the epidemic and it can be divided into five grades. We assign $k$ respectively as $0.1,0.15,0.2,0.25,0.5$, and corresponding dose level for the required area are $5,4,3,2,1$. If everyone can get treatment, then

$$
\sum_{i=1}^{n} w_{i} \geq A
$$

We obtained that the number of infected people at the moment ${ }^{t}$ is $I(t)$ [10]. Set the average drug quantity of each patient needed per week to be $\tau$.Under the influence of the evaluation coefficient $\xi$, the total quantity needed weekly is: $A=\tau \cdot \xi \cdot I(t)$

Assuming that all the quantity is enough, and each service center can provide the quantity $g_{j}$, and 
the ideal situation is:

$$
g_{j} \geq \frac{A}{T}
$$

Set shipments by the service center $P_{j}\left(x_{j}, y_{j}\right)$ to the isolation $Q_{i}\left(x_{i}, y_{i}\right)$ is $c_{i j}$. Considering the number of drugs, the increase of drug attrition rate in transport distance, and the increase of vaccine preservation costs, we take the destruction coefficient ${ }^{p}$ of the drug rate into account. We try best to find a way to make the drug loss as less as possible so that to get the minimum cost of transportation. The goal and constraint condition are set up as follows:

$$
\begin{aligned}
& \min z=p T \sum_{i=1}^{n} \sum_{j=1}^{T} c_{i j} \sqrt{\left(x_{j}-x_{i}\right)^{2}+\left(y_{j}-y_{i}\right)^{2}} \\
& \text { s.t. }\left\{\begin{array}{l}
\sum_{i=1}^{n} c_{i j} \leq g_{j}, j=1,2, \cdots, T \\
\sum_{j=1}^{T} c_{i j} \geq w_{j}, i=1,2, \cdots, n
\end{array}\right.
\end{aligned}
$$

Take the corresponding parameters for different service center number, get the result:

Table 1 Different optimal values corresponding to the value of $T$

\begin{tabular}{ccccccccccc}
\hline$T$ & 7 & 8 & 9 & 10 & 11 & 12 & 13 & 14 & 15 & 20 \\
\hline$z$ & 66.69 & 66.18 & 65.89 & 65.52 & 65.91 & 72.16 & 78.51 & 79.01 & 80.15 & 109.08 \\
\hline
\end{tabular}

As can be seen from the table, if $T$ is 10 , the target is minimum .With the increase of $\mathrm{T}$ by following 20 , the objective function values will also growing. So, the number of the best service center is 10 .

MATLAB output coordinate results:
$(0.550,0.453)$;
$(0.753,0.483)$
$(0.440,0.663)$
$(0.493,0.564)$
$(0.900,0.210)$
$(0.653,0.448)$;
$(0.627,0.800)$;
$(0.825,0.672)$;
$(0.825,0.672)$;
$(0.713,0.653)$;

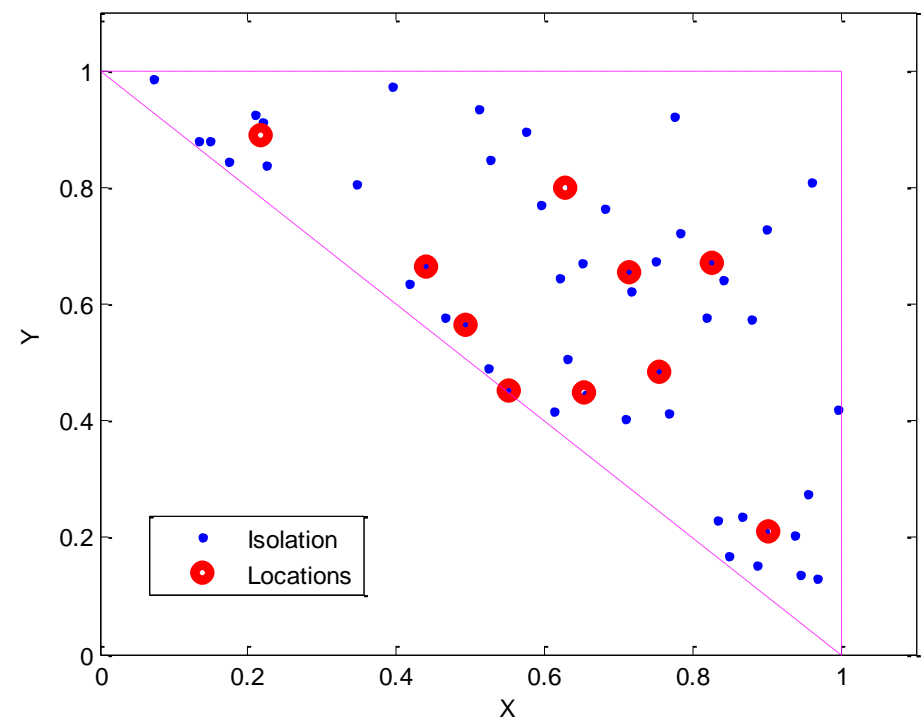

Figure. 1 The distribution of the isolation and the service center locations 
On the map is expressed as:

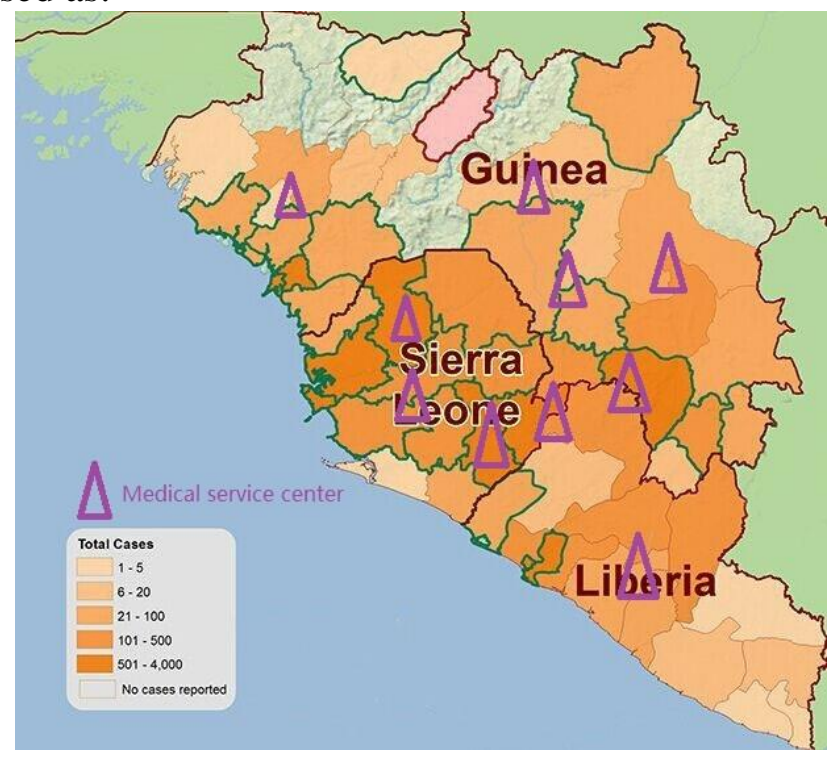

Figure. 2 The distribution of the service center locations

It is concluded that most of the drug service centers are distributed in the three countries. Combined with the actual problem, the illness in the central part is serious, and the city is relatively dense, marginal condition is relatively light, so the calculation of number in the center area of the service center is relatively more, and the border is less, in line with the actual.

The results are based on the assumption that the dose of medicine is enough for all of the patients' curing. If $g_{j}{ }^{\prime}<g_{j}$, means that not all the patients can be cured and we can't achieve a thorough result.

\section{System estimation}

First of all, on the basis of the coverage problem in graph theory, we can conclude the minimum number of each distribution station roughly. Then combining the location problem, we build objective function and constraint condition so as to solve. It is concluded that the optimal number of service centers is 10, and the position is also consistent with the actual problem. The accuracy of model is higher.

\section{System optimization}

The drug allocation amount for each service center is the same in this model, which is a little bit not tally with the reality, so that the ${ }^{g_{j}}$ of each service center is not necessarily equal. In the improvement of the service center, we add a weight to the required quantity of the service center, and the weight is divided into three levels. Keeping the objective function and constraint conditions constant, finally we get the target function of minimum value: $z_{\min }{ }^{\prime}=64.42$. Compared with the previous optimization $z_{\min }=65.52$, the corresponding service center location in the picture at this time as follows: 


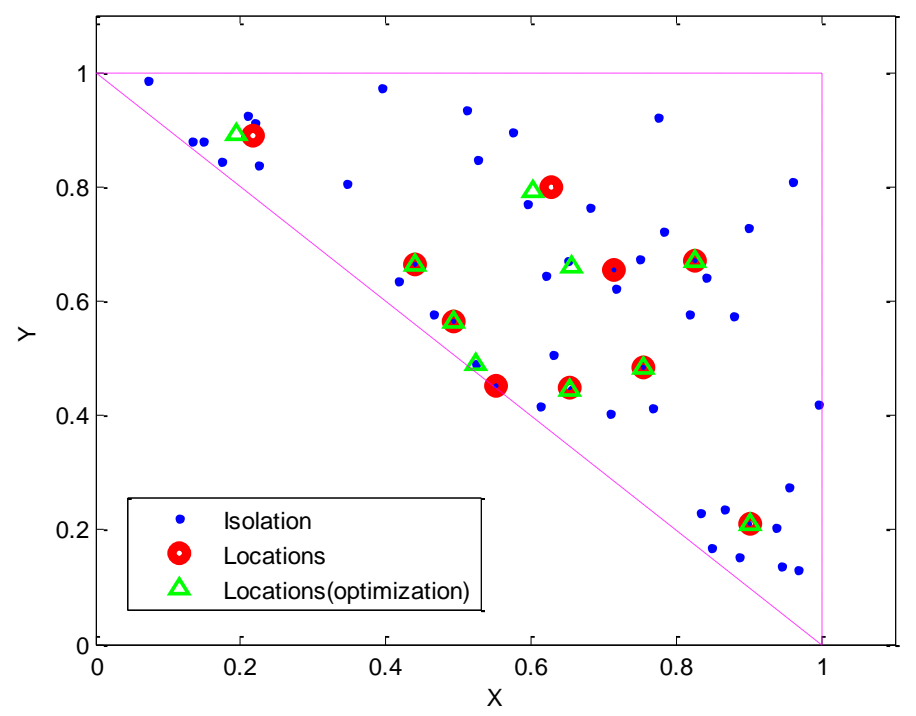

Figure. 3 Service center location in different weight

Analysis from the picture shows that some service areas coincide with the original after optimization, but there is also a part of the adjustment. Combined with the target value, the effect is significant after optimization. So on the drug content allocation of each service area has some room for improvement.

\section{References}

[1]S. X: Wireless sensor network Repeaters placed problem and algorithm research (Ph.D., Hangzhou Dianzi University, China 2009)

[2]Y.H. Pu: Graph theory and its application (Southeast university press, China 2002)

[3]http://zh.wikipedia.org/wiki/\%E9\%9D\%9E\%E6\%B4\%B2\#.E5.9C.B0.E7.90.86

[4]X.S. Yuan, D.H. Shao and S.L. Yu: The application of LINGO and Excel in mathematical modeling, (Science press, China 2007). (In Chinese)

[5]J. Pinter: Continuous Global Optimization: Models, Algorithms and Software (Encyclopedia of Optimization, 2001), p.328

[6]H.Y. Liu, Z.P. Li and H.Z. Ye: Logistics distribution center location model. Journal of southwest jiaotong university. Vol.35(2000) No.3, p.311

[7]X.F. Tang, H.J. Mao and X.H. Li: Journal of southeast university (natural science edition), Vol. 39 (2009) No.2, p.404

[8]S.K. Si and X.J. Sun: Mathematical modeling algorithm and application (National Defense Industry Press, China 2014), p.14 (In Chinese)

[9]Data of WHO. Feb 2015, Web site : http://www.who.int/mediacentre/factsheets/fs103/en/

[10]Q.Y. Jiang, J.X. Xie and J. Ye: Mathematical model (Higher Education Press, China 2003), p. 135 (In Chinese) 